\title{
Deep-Collapse Operation of Capacitive Micromachined Ultrasonic Transducers
}

\author{
Selim Olcum, Member, IEEE, F. Yalcin Yamaner, Student Member, IEEE, \\ Ayhan Bozkurt, Member, IEEE, and Abdullah Atalar, Fellow, IEEE
}

\begin{abstract}
Capacitive micromachined ultrasonic transducers (CMUTs) have been introduced as a promising technology for ultrasound imaging and therapeutic ultrasound applications which require high transmitted pressures for increased penetration, high signal-to-noise ratio, and fast heating. However, output power limitation of CMUTs compared with piezoelectrics has been a major drawback. In this work, we show that the output pressure of CMUTs can be significantly increased by deep-collapse operation, which utilizes an electrical pulse excitation much higher than the collapse voltage. We extend the analyses made for CMUTs working in the conventional (uncollapsed) region to the collapsed region and experimentally verify the findings. The static deflection profile of a collapsed membrane is calculated by an analytical approach within $\mathbf{0 . 6 \%}$ error when compared with static, electromechanical finite element method (FEM) simulations. The electrical and mechanical restoring forces acting on a collapsed membrane are calculated. It is demonstrated that the stored mechanical energy and the electrical energy increase nonlinearly with increasing pulse amplitude if the membrane has a full-coverage top electrode. Utilizing higher restoring and electrical forces in the deep-collapsed region, we measure 3.5 MPa peak-to-peak pressure centered at $6.8 \mathrm{MHz}$ with a $106 \%$ fractional bandwidth at the surface of the transducer with a collapse voltage of $35 \mathrm{~V}$, when the pulse amplitude is $160 \mathrm{~V}$. The experimental results are verified using transient FEM simulations.
\end{abstract}

\section{INTRODUCTION}

$\mathrm{C}$ APacitive micromachined ultrasonic transducers $\mathcal{U}$ (CMUT) have been introduced as a promising technology especially for medical imaging applications [1]-[3]. CMUTs are micromachined electrostatic transducers with parallel plate structures with a moving top electrode and a rigid substrate electrode. More than 100\% fractional bandwidth can be easily achieved when they are loaded with a liquid medium [4]. Integrated circuit manufacturing technology enables CMUTs to be produced in different sizes and shapes using basic lithography techniques. The difficulties in the fabrication processes have been gradually solved and several different approaches have been

Manuscript received August 6, 2010; accepted July 28, 2011. This work has been supported by the Scientific and Technological Research Council of Turkey (TUBITAK) under project grants 104E067, 105E023, and 107T921. S. Olcum gratefully acknowledges TUBITAK and Aselsan A.S. for their Graduate Scholarship and Fellowship Programs. A. Atalar acknowledges the support of the Turkish Academy of Sciences (TUBA).

S. Olcum and A. Atalar are with Bilkent University, Electrical and Electronics Engineering Department, Ankara, Turkey (e-mail: selim@ ee.bilkent.edu.tr).

Y. Yamaner and A. Bozkurt are with Sabanci University, Electronics Engineering, Istanbul, Turkey.

Digital Object Identifier 10.1109/TUFFC.2011.2104 proposed during the last decade [5]-[7]. Large numbers of CMUTs can be produced on the same wafer, resulting in reduced costs compared with their piezoelectric alternatives. In addition, CMUT technology can be used to manufacture transducer arrays integrated with driving electronic circuits. Recently, 2-D CMUT arrays with fully integrated electronics have been demonstrated in challenging medical imaging applications [8]-[10]. In many applications, such as intravascular ultrasound (IVUS), 2-D arrays, or flexible arrays, for which piezoelectrics seem to be inadequate, CMUTs have provided promising results [9]-[12].

Ultrasound imaging applications require high transmitted pressures for increased penetration and better signalto-noise ratio. For therapeutic applications, higher output pressures are necessary for faster heating of the tissue. However, output power limitations of CMUTs compared with piezoelectrics have been the greatest drawback since they were first introduced. During the past decade, several attempts have been made to increase the power output of CMUTs. Collapse and collapse-snap-back modes of operation have boosted the pressure output considerably [13] [15]. Dual-electrode structures introduced by Güldiken et al. [16] also improved the pressure output of the CMUTs by increasing the electromechanical coupling coefficient. It has been demonstrated that use of rectangular membranes increases the fill-factor and hence the output pressure [17]. These improvements in the output power capability of CMUTs make them good candidates for medical imaging and high-intensity focused ultrasound (HIFU) applications. The first attempts and feasibility results using CMUTs as a HIFU transducer have been reported recently [18], [19].

CMUTs have been widely studied in terms of equivalent circuit modeling [20]-[23] and finite element modeling (FEM) simulations [24], [25]. However, analytical formulations and equivalent circuit models have been developed only for the conventional (uncollapsed) regime of operation [26]-[28]. The collapsed mode of operation has been investigated in terms of FEM simulations [29], but lacks accurate analytical models for understanding the mechanics and the limits of the mode.

In this work, we show that the output pressure of a CMUT can be increased considerably by electrically exciting it far beyond the collapse point. We extend the analyses made for CMUTs working in the uncollapsed region to the collapsed region to understand the dynamics under these conditions. The electrical and restoring forces acting on a collapsed membrane are calculated. We test our 
analytical results with FEM simulations and experiments performed on fabricated CMUTs.

\section{Mechanics of CMUTS}

The radiated pressure output from a CMUT when it is excited by an electrical pulse is related to the forces acting on the membrane during the pulse cycle. During any unipolar pulse excitation, the membrane movement can be investigated in two parts: collapse (deflect) and release. In the following analysis, we investigate the electrical and mechanical forces acting on a CMUT membrane during the collapse and release parts of a pulse cycle. The static membrane deflection profile at a dc bias point is required to determine the electrical and mechanical forces acting on the membrane.

\section{A. Uncollapsed Deflection Profile}

The expression for the deflection, $x$, of a clamped circular membrane as a function of radius, $r$, under uniform pressure was derived by Timoshenko [30, p. 55] as

$$
x(r)=\frac{P}{64 D}\left(a^{2}-r^{2}\right)^{2}
$$

where $P$ is the applied uniform pressure and $a$ is the radius of the membrane. The flexural rigidity, $D$, is defined as

$$
D \equiv \frac{E t_{\mathrm{m}}^{3}}{12\left(1-\nu^{2}\right)}
$$

where $E$ is the Young's modulus, $\nu$ is the Poisson's ratio, and $t_{\mathrm{m}}$ is the thickness of the membrane. A more general approach for the deflection profile of circular plates is discussed in [31]. The validity and accuracy of (1) for the conventional regime of CMUTs are demonstrated in a previous work [26].

\section{B. Collapsed Deflection Profile}

A different set of boundary conditions should be utilized for the calculation of the profile when the center of the membrane touches the substrate (Fig. 1). We use the general solution for the deflection of a uniformly loaded collapsed circular plate derived by Timoshenko [30, p. 309] as the starting point:

$$
\begin{aligned}
x(r)= & C_{1}+C_{2} \ln r+C_{3} r^{2}+C_{4} r^{2} \ln r+\frac{r^{4}}{64 D} P \\
& \text { for } b \leq r \leq a,
\end{aligned}
$$

where $b$ is the contact radius.

We apply boundary conditions to (3), which characterize the deflected shape of a collapsed and clamped membrane:

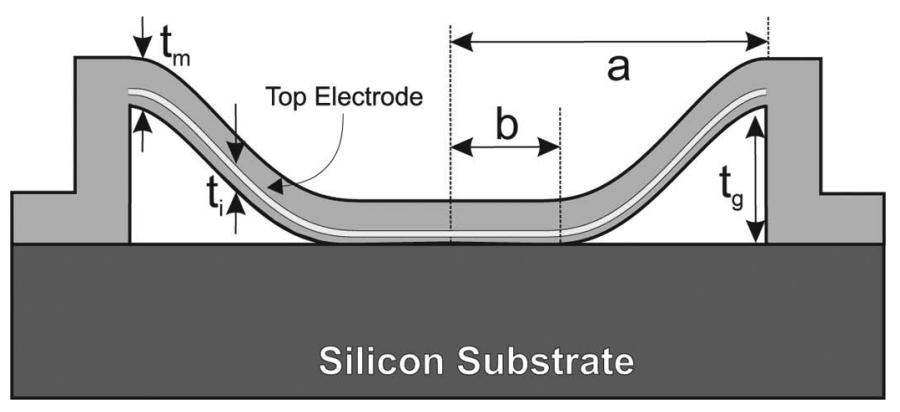

Fig. 1. Cross section of a collapsed clamped circular membrane with radius $a$, thickness $t_{\mathrm{m}}$, and gap height of $t_{\mathrm{g}}$. The top electrode is at a distance $t_{\mathrm{i}}$ above from the gap. The contact radius is $b$.

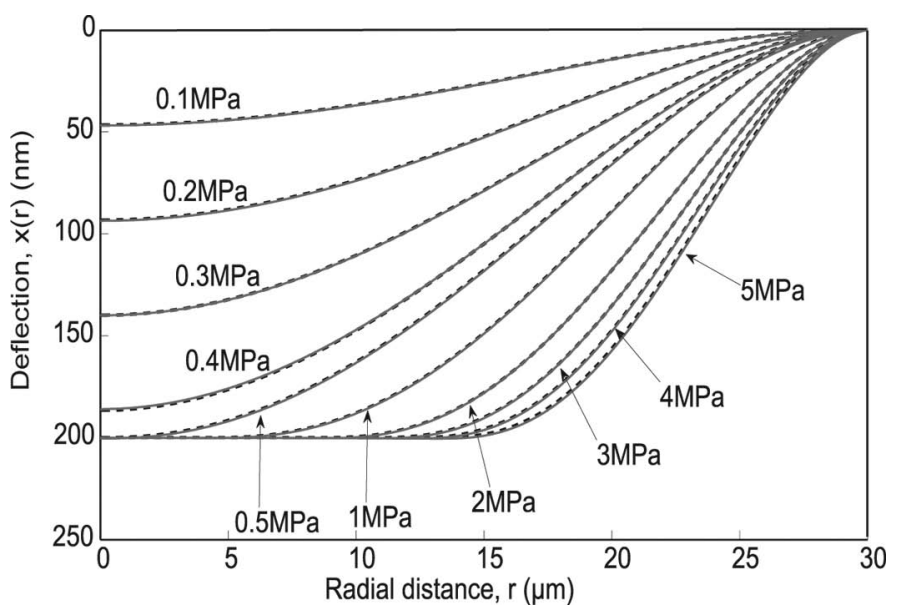

Fig. 2. Comparison of deflection profiles obtained by (1) and (3) (solid curves) with mechanical FEM simulations (dashed curves). Values of the uniform pressure applied on the membrane are indicated for each curve in the figure. The physical parameters of the CMUT under consideration are given in Table I.

$$
\begin{gathered}
x(a)=0, \quad x(b)=t_{\mathrm{g}}, \\
\left.\frac{\mathrm{d} x(r)}{\mathrm{d} r}\right|_{r=a}=0,\left.\quad \frac{\mathrm{d} x(r)}{\mathrm{d} r}\right|_{r=b}=0, \\
M_{\mathrm{r}}(b)=-\left.D\left(\frac{\mathrm{d}^{2} x(r)}{\mathrm{d} r^{2}}+\frac{\nu}{r} \frac{\mathrm{d} x(r)}{\mathrm{d} r}\right)\right|_{r=b}=0,
\end{gathered}
$$

where $t_{\mathrm{g}}$ is the gap between the membrane and the substrate and $M_{\mathrm{r}}$ is the radial bending moment on the membrane. The contact radius, $b$, is determined by solving (6) in terms of the four unknown constants of (3), which in turn are determined using the four boundary conditions of (4) and (5). The deflection profiles of (1) and (3) are plotted in Fig. 2 (solid) along with ANSYS simulation results (dashed) for different applied uniform pressures.

In the following analysis, we use the average displacement, $x_{\mathrm{a}}$, as the lumped displacement measure of a circular clamped membrane:

$$
x_{\mathrm{a}} \equiv \frac{1}{\pi a^{2}} \int_{0}^{a} 2 \pi r x(r) \mathrm{d} r .
$$


TABle I. Physical Dimensions of the CMUT Element.

\begin{tabular}{ll}
\hline Physical dimensions & \\
\hline Radius, $a$ & $30 \mu \mathrm{m}$ \\
Electrode radius & $30 \mu \mathrm{m}$ \\
Membrane thickness, $t_{\mathrm{m}}$ & $1.4 \mu \mathrm{m}$ \\
Top electrode thickness & $120 \mathrm{~nm}$ \\
Gap height, $t_{\mathrm{g}}$ & $0.2 \mu \mathrm{m}$ \\
Insulator thickness, $t_{\mathrm{i}}$ & $0.4 \mu \mathrm{m}$ \\
Cell-to-cell separation & $5 \mu \mathrm{m}$ \\
Number of cells in an element & 121 \\
Size of an array element & $0.71 \times 0.71 \mathrm{~mm}$ \\
\hline
\end{tabular}

For the uncollapsed case, the average displacement is related to the applied pressure, $P$, linearly as given by [26] and [28]:

$$
x_{\mathrm{a}}=\frac{a^{4}}{192 D} P \text {. }
$$

For the collapsed case, the average displacement can be written as

$$
x_{\mathrm{a}}=\frac{b^{2}}{a^{2}} t_{\mathrm{g}}+\frac{2}{a^{2}} \int_{b}^{a} r x(r) \mathrm{d} r .
$$

To simplify the expression in (9), we define $X_{\mathrm{a}}(r)$ using a dummy variable $\rho$ :

$$
X_{\mathrm{a}}(r) \equiv \frac{2}{a^{2}} \int_{0}^{r} \rho x(\rho) \mathrm{d} \rho .
$$

We find, using (3),

$$
\begin{aligned}
X_{\mathrm{a}}(r)= & \frac{r^{2}}{a^{2}}\left(C_{1}+\frac{C_{2}}{2}(2 \ln r-1)+\frac{C_{3} r^{2}}{2}\right. \\
& \left.+\frac{C_{4} r^{2}}{2}\left(\ln r-\frac{1}{4}\right)+\frac{r^{4}}{192 D} P\right) .
\end{aligned}
$$

Hence, from (9), we find the nonlinear relationship between the average displacement and the applied pressure as

$$
x_{\mathrm{a}}=\frac{b^{2}}{a^{2}} t_{\mathrm{g}}+X_{\mathrm{a}}(a)-\frac{b^{2}}{a^{2}} X_{\mathrm{a}}(b) .
$$

\section{Restoring Force of the Membrane}

Because the applied pressure is balanced by the restoring force of the membrane, we can find the restoring force easily. The restoring force as a function of average displacement is calculated by changing the uniform pressure, $P$. The amplitude of the restoring force at each pressure value is calculated by simply multiplying the applied pressure by the membrane area. Analytical relations in (1) and (3) are used for calculation of membrane profiles, whereas (8) and (12) are used to find the average displacement. This highly nonlinear restoring force curve is plotted (solid line) in Fig. 3 as a function of average displacement, $x_{\mathrm{a}}$.

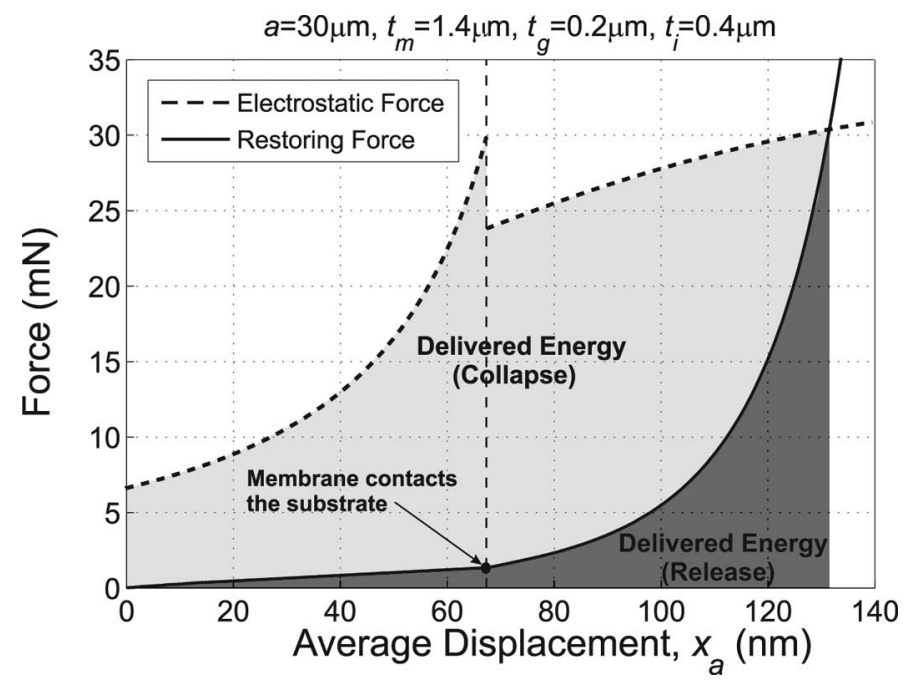

Fig. 3. The electrical force (dashed curve) at a bias of $200 \mathrm{~V}$ and the mechanical restoring force (solid curve) as a function of average displacement, $x_{\mathrm{a}}$.

\section{Electrical Force on the Membrane}

The electrical force can be determined using the same deflection profile by calculating the change in the stored electrical energy. The electrical force acting on a membrane at a given average displacement, $x_{\mathrm{a}}$, with a constant bias voltage of $V$ can be calculated from [26] or [28]:

$$
F_{\mathrm{e}}\left(x_{\mathrm{a}}\right)=\frac{\mathrm{d} E_{\mathrm{s}}}{\mathrm{d} x_{\mathrm{a}}}=\frac{1}{2} \frac{\mathrm{d} C\left(x_{\mathrm{a}}\right)}{\mathrm{d} x_{\mathrm{a}}} V^{2},
$$

where $E_{\mathrm{s}}$ is the stored electrical energy and $C\left(x_{\mathrm{a}}\right)$ is the total capacitance of a CMUT which can be found from

$$
C\left(x_{\mathrm{a}}\right)=\int_{0}^{a} \frac{2 \pi \varepsilon_{0} r}{t_{\mathrm{g}}^{\prime}-x(r)} \mathrm{d} r
$$

where $t_{\mathrm{g}}^{\prime}=t_{\mathrm{g}}+t_{i} / \varepsilon_{r}$ is the effective gap height.

Obviously, for a membrane deflected by a dc bias, the charge distribution and, hence, the electrostatic force over the membrane, is not uniform and is a strong function of radius. The magnitude of the electrostatic force depends heavily on the radial position. But the deflection profile is mainly determined by the total electrostatic force, rather than how this force is distributed over the membrane. Therefore, we assume that any deflection profile caused by an applied voltage can be approximated by the deflection resulting from a uniform pressure generating an equal total force [26]. This is a good assumption, especially when the membrane has an electrode covering its full surface.

The variation in the total electrical force with respect to the average displacement is plotted as the dashed curve in Fig. 3, for a dc bias of $200 \mathrm{~V}$. The electrical force is discontinuous at the point of contact, because of discontinuity of the derivative of the capacitance with respect to average displacement. The intersection point of the electrical force with the restoring force in Fig. 3 gives the 


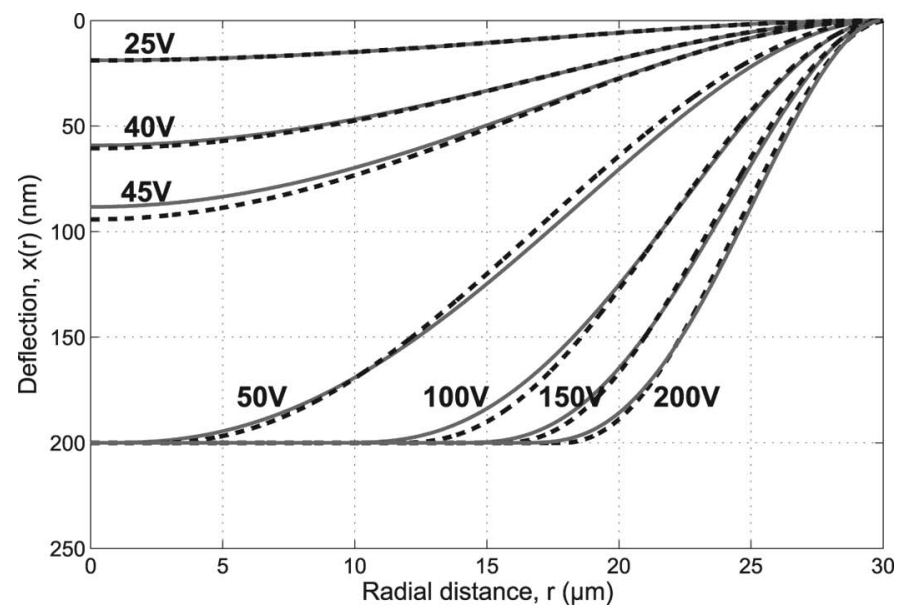

Fig. 4. Comparison of deflection profiles of CMUT membranes under dc bias, obtained by (1) and (3) (solid curves), with coupled electrostaticmechanic FEM simulations (dashed curves). Values of the applied dc bias on the membrane are indicated for each curve on the figure. The physical parameters of the CMUT under consideration are given in Table I.

equilibrium average displacement point for an applied bias on the CMUT of $200 \mathrm{~V}$. In this case, the average displacement is found to be $132.7 \mathrm{~nm}$, which compares well with the FEM simulation result of $133.7 \mathrm{~nm}$. The root mean square error between the profiles of the analytical result and the electromechanical FEM simulation is $0.6 \%$.

In Fig. 4, the deflection profiles of the membrane obtained by the electromechanical FEM analyses (dashed) when the membrane is excited with different dc voltages are depicted along with the profiles calculated by the analytical expression in (1) and (3) (solid). The total force over the CMUT membrane as a result of the uniform pressure used in the equations is equal to the total force generated by the dc voltage.

\section{E. Energy Delivered to the Medium}

The mechanical energy stored by the membrane is released to the immersion medium when the voltage across the electrodes is zero. The stored energy can be calculated by integrating the restoring force curve to find the area of the dark shaded region of Fig. 3, and is found to be $0.63 \mathrm{~nJ}$ for the CMUT of interest. The average displacement and the mechanical restoring force have a linear relation in the uncollapsed region. When the membrane touches the substrate, the relation becomes highly nonlinear and the amount of energy stored in the membrane increases faster than in the uncollapsed region.

When a high voltage is applied across the CMUT electrodes, a part of the input electrical energy is stored as the mechanical energy, while another part is delivered to the immersion medium. Because the net force applied to the medium is the difference between the electrical and the restoring force curves, the energy transferred to the medium can be found by calculating the area between the two curves. This area corresponding to an energy of $2.0 \mathrm{~nJ}$ is shown as the light shaded region in Fig. 3.

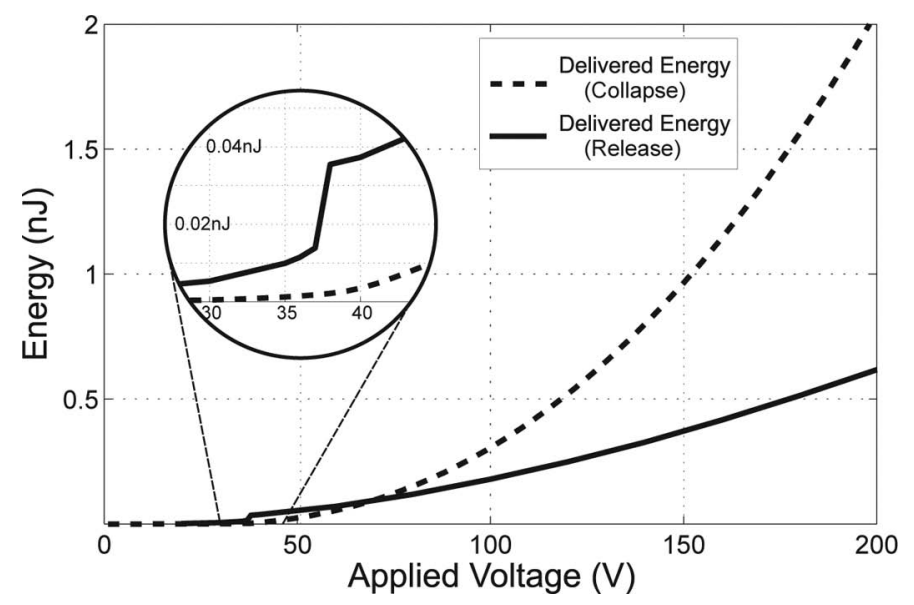

Fig. 5. Delivered energy during the release (solid curve) and collapse (dashed curve) parts of a unipolar pulse cycle. CMUT parameters: $a=$ $30 \mu \mathrm{m}, t_{\mathrm{m}}=1.4 \mu \mathrm{m}, t_{\mathrm{g}}=0.2 \mu \mathrm{m}$, and $t_{\mathrm{i}}=0.4 \mu \mathrm{m}$.

The energies delivered to the medium during the collapse and the release periods are shown in Fig. 5 as two separate curves as a function of applied voltage. Clearly, more energy is delivered during the collapse period than in the release period. The difference increases as the applied voltage is increased further.

The effects of the collapse and collapse/snap-back modes on the radiated energy are striking, as pointed out in previous studies [13]-[15], [32]. Moreover, increasing the voltage beyond the collapse voltage increases the radiated energy considerably in both the collapse and release periods. We call this mode deep-collapse operation, where the nonlinear forces are utilized during the transmission cycle, well beyond the collapse point. We should note that this mode is effective if we have a full-coverage top electrode. For the case of a partial electrode, the membrane cannot store any more energy once the contact radius ( $b$ in Fig. 1 ) is equal to the electrode radius.

\section{EXPERIMENTAL RESUlts}

\section{A. Fabrication of CMUTs}

We use a surface microfabrication technology similar to that described in [7] utilizing a silicon nitride dielectric deposited by a plasma-enhanced chemical vapor deposition (PECVD) system. The process conditions used in the PECVD reactor are given in Table II, along with some material properties of the film ${ }^{1}$. The physical parameters of the fabricated CMUT element are listed in Table I.

The microfabrication process is performed on a low resistivity $(<0.005 \Omega-\mathrm{cm}), \sim 330 \mu \mathrm{m}$ thick silicon wafer, which constitutes the bottom electrode. Over the surface of the bare silicon wafer, 200-nm-thick $\left(=t_{\mathrm{g}}\right)$ sputtered chromium is used as the sacrificial layer, which determines the gap underneath the membrane. After photolithograph-

\footnotetext{
${ }^{1}$ The leakage current measurement is performed using a Keithley 2100 sourcemeter (Keithley Instruments Inc., Cleveland, OH).
} 
TABLE II. Low-Stress Silicon Nitride Deposition Conditions.

\begin{tabular}{ll}
\hline Deposition conditions & \\
\hline Temperature & $250^{\circ} \mathrm{C}$ \\
Power & $9 \mathrm{~W}$ \\
Pressure & $1000 \mathrm{mTorr}$ \\
$\% 2 \mathrm{SiH}_{4}$ in $\mathrm{H}_{2}$ & $200 \mathrm{sccm}$ \\
$\mathrm{NH}_{3}$ & $4 \mathrm{sccm}$ \\
$\mathrm{He}$ & $50 \mathrm{sccm}$ \\
$\mathrm{N}_{2}$ & $35 \mathrm{sccm}$ \\
Deposition speed & $\sim 8.3 \AA / \mathrm{s}$ \\
Intrinsic stress & $\sim 25 \mathrm{MPa}$ tensile \\
Relative permittivity, $\varepsilon_{\mathrm{r}}$ & $\sim 5.4$ \\
Leakage current of $400-\mathrm{nm}$ film & 0.5 to $1 \mathrm{nA}$ at $100 \mathrm{~V}$ \\
\hline
\end{tabular}

ic patterning, 400-nm $\left(=t_{\mathrm{i}}\right)$ silicon nitride is deposited as the insulation layer between the electrodes. The top electrode is made from a thermally evaporated gold layer with titanium on both sides for good adhesion. Following the lift-off and cleaning steps, another 400-nm layer of silicon nitride is deposited on top of the metal electrode to bury the electrode. To allow the etchants to reach the sacrificial layer during the release step, small holes are drilled in the silicon nitride layer using a reactive ion etching (RIE) reactor. Following the release step involving a chrome etchant, the etch holes are sealed by depositing a final 600-nm-thick layer of silicon nitride, bringing the final thickness of the silicon nitride membrane to $1.4 \mu \mathrm{m}$ (= $\left.t_{\mathrm{m}}\right)$. Finally, the silicon nitride layer over the bond-pads is etched away using the RIE reactor.

Fig. 6 shows a micrograph of the fabricated CMUT element consisting of $11 \times 11$ cells of $30-\mu$ m-radius membranes. Each cell has 5 - $\mu \mathrm{m}$ spacing with the neighboring cells, resulting in a fill factor of approximately $67 \%$. The cells are measured to have an uncollapsed natural resonance frequency of $5.3 \mathrm{MHz}$ in air and a collapse voltage of approximately $35 \mathrm{~V}$.

\section{B. Experiments}

Immersion experiments are carried out in an oil-filled tank (see Fig. 7) using CMUT elements of $0.71 \times 0.71 \mathrm{~mm}$ as the acoustic source and a calibrated hydrophone $1 \mathrm{~cm}$ away as the receiver. To find the pressure at the surface of the CMUTs, diffraction and attenuation ${ }^{2}$ losses are compensated using the formulations in [34, ch. 3] and [8], respectively.

40-ns-long pulses with $5 \mathrm{~V}$ amplitude are applied to the CMUT element while the DC bias is varied to measure the small signal transmit sensitivity of the device. The measured peak-to-peak pressures are plotted in Fig. 8. A small signal transmit sensitivity of more than $40 \mathrm{kPa} / \mathrm{V}$ is measured using a 95-V bias as compared with $20 \mathrm{kPa} / \mathrm{V}$ at the edge of snap-back. With a partial electrode coverage, e.g., $50 \%$ coverage [14], the transmitted pressure amplitude saturates after the electrode region of the membrane fully

\footnotetext{
${ }^{2}$ For sunflower oil $\alpha=5.68 \times 10^{-12} \mathrm{~m}^{-1} \cdot \mathrm{Hz}^{-1.85}[28]$.
}

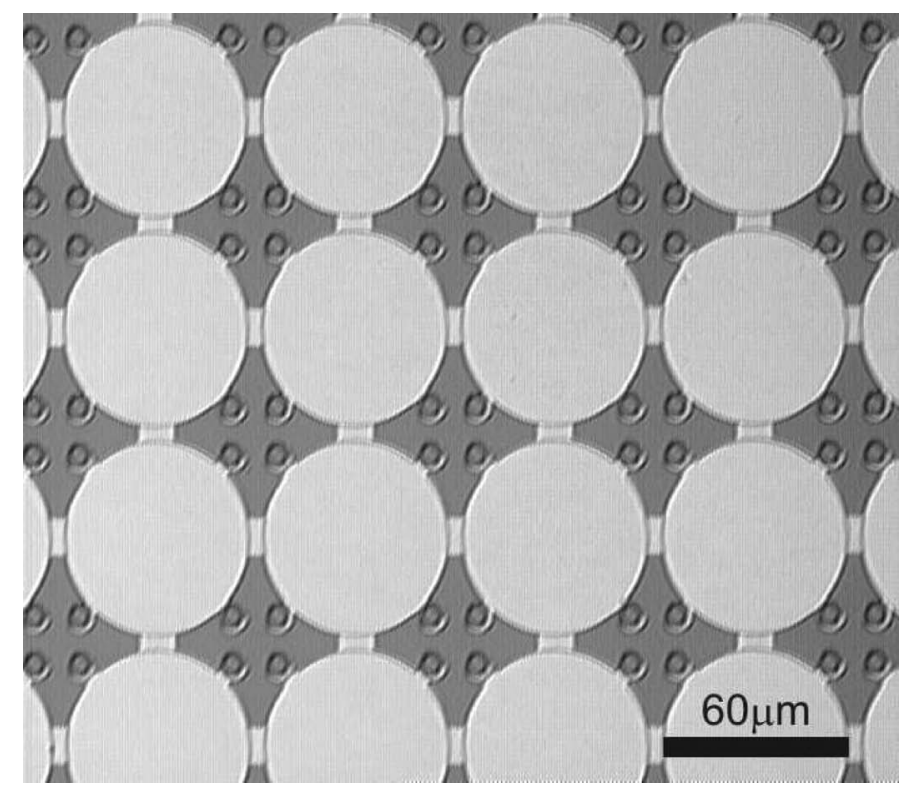

Fig. 6. An optical micrograph of a fabricated CMUT element: 121 cells (11 $\times 11)$, each with a radius of $a=30 \mu \mathrm{m}$ and a cell-to-cell separation of $5 \mu \mathrm{m}$. The aperture size of the element is $0.71 \times 0.71 \mathrm{~mm}$ and the fill factor of the cells is $67 \%$.

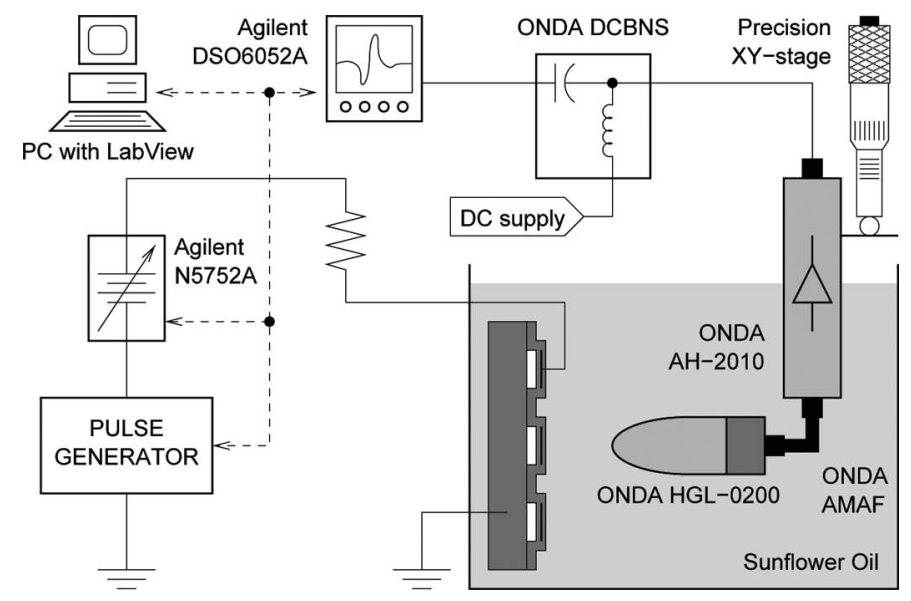

Fig. 7. A schematic diagram of the experimental setup used during the transmission experiments. A calibrated hydrophone (HGL-0200, Onda Corp., Sunnyvale, CA) with a preamplifier (AH-2010, Onda Corp.) is used for recording the transmitted pressure waveform.

collapses to the substrate. Increasing the voltage further does not provide more attraction force on the membrane.

For testing the deep-collapse operation, 40-ns-long pulses with different negative amplitudes are applied to the CMUTs. The applied bias voltage is changed and the negative pulse amplitude is always kept equal to the dc bias. Transmitted peak-to-peak pressure values are measured and plotted as a function of the applied pulse amplitude in Fig. 9.

To determine the pressure output theoretically, we must solve the dynamic problem involving the membrane mass, the radiation impedance of the immersion medium, and the nonlinear spring constant of the membrane excited by a position-dependent force. We solved this nonlinear 


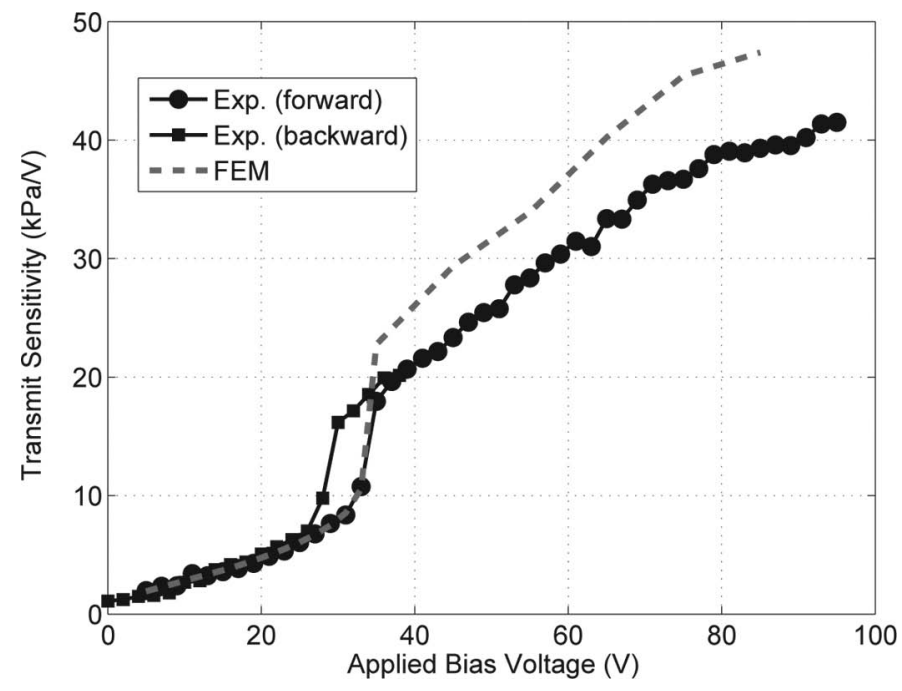

Fig. 8. Measured small-signal transmit sensitivity in terms of peak-topeak pressure per applied voltage at the surface of the CMUTs. CMUTs are excited by a 40-ns-long, 5-V pulse while the bias is monotonically increased (circles) and monotonically decreased (squares).

problem in the time domain using our FEM model (see the Appendix). In FEM simulations, the loading medium is defined as a water column in a rigid baffle. This would model the medium correctly for a CMUT at the center region of a large array of cells. It was shown in [35] that the average radiation resistance seen by an array of CMUT cells is approximately one-half of the radiation resistance seen by the center cell. Because the CMUT element in the experiments has a finite size, the difference between the FEM simulations and the experimental results can partially be attributed to the inaccurate modeling of the liquid loading. Moreover, the FEM model assumes a perfectly rigid substrate, preventing any energy loss to the substrate. This may not be the case in reality, especially during the collapsed state.

The superiority of full electrode coverage over partial coverage can be seen in the FEM simulations of Fig. 9. The pressure generated by CMUTs with half-electrodes saturate, however CMUTs with full electrode coverage can still respond to increased pulse amplitude.

The measured pressure waveform when the CMUTs are excited by a negative $160-\mathrm{V}$ pulse on top of $160-\mathrm{V}$ bias is depicted in Fig. 10 (solid line) along with the waveform obtained with an FEM simulation (dashed line) performed for a cell at the center of an element. During experiments, a peak-to-peak pressure of $3.5 \mathrm{MPa}$ is measured at the surface of the CMUTs using this excitation. The measured waveform compares well with the FEM simulations. The positive cycle of the pressure waveform has a smaller amplitude and lasts longer, whereas the negative cycle is larger in amplitude and faster in the time axis. The transmitted pressure waveform in Fig. 10 has a fractional bandwidth of $106 \%$ centered at $6.8 \mathrm{MHz}$, which is depicted in Fig. 11. The notch at $12.5 \mathrm{MHz}$ in the spectrum of the experimental result is due to the energy coupling to the substrate mode. FEM simulations do not predict the sub-

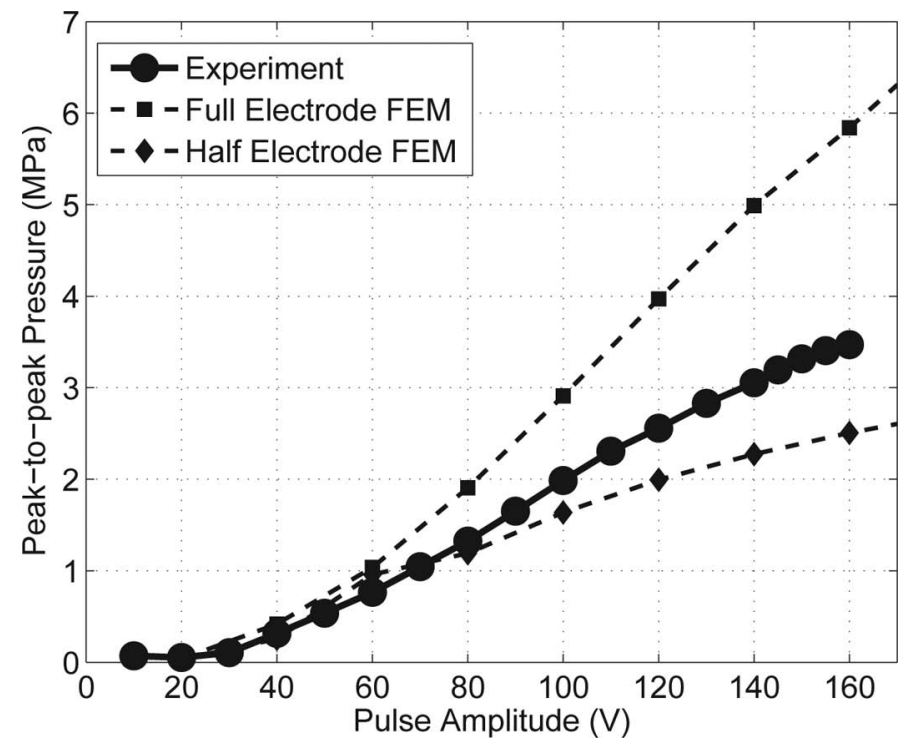

Fig. 9. Measured peak-to-peak pressure output at the surface of full-electrode CMUTs (solid line) when excited with a pulse of varying negative amplitude on top of an equal amplitude bias. FEM simulation results (dashed lines) for full and half-electrode CMUTs are shown for comparison.

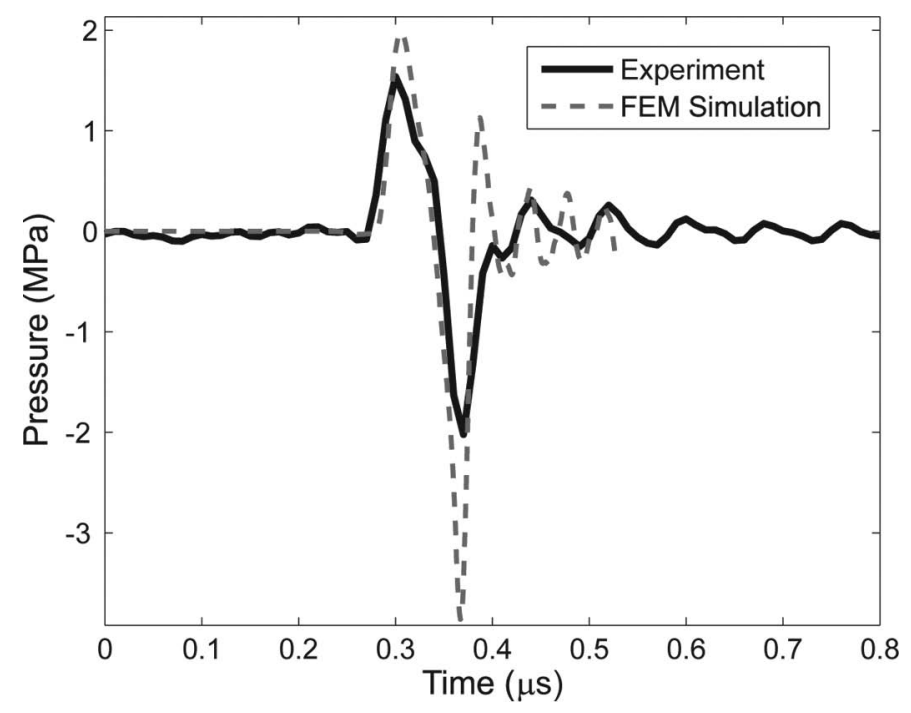

Fig. 10. Measured (solid line) and simulated (dashed line) pressure waveforms at the surface of the CMUTs when the transducers are excited with a 40-ns-long, negative $160-\mathrm{V}$ pulse on top of $160-\mathrm{V}$ bias. The experiments and simulations are performed on the CMUT with the physical parameters given in Table I.

strate mode because we only model the membrane and the stand region of a CMUT. We observe that the spectrum of the FEM simulation is centered at a higher frequency with a larger fractional bandwidth. The center cell sees a larger radiation resistance and a smaller radiation reactance [35], which would generate a larger bandwidth and a higher center frequency.

In this paper, all tested CMUTs have full electrode coverage on their membranes. The full electrode coverage maintains high mechanical and electrical forces well above the collapse voltage. A half-electrode coverage, which is optimal [36] for the uncollapsed operation, is not prefer- 


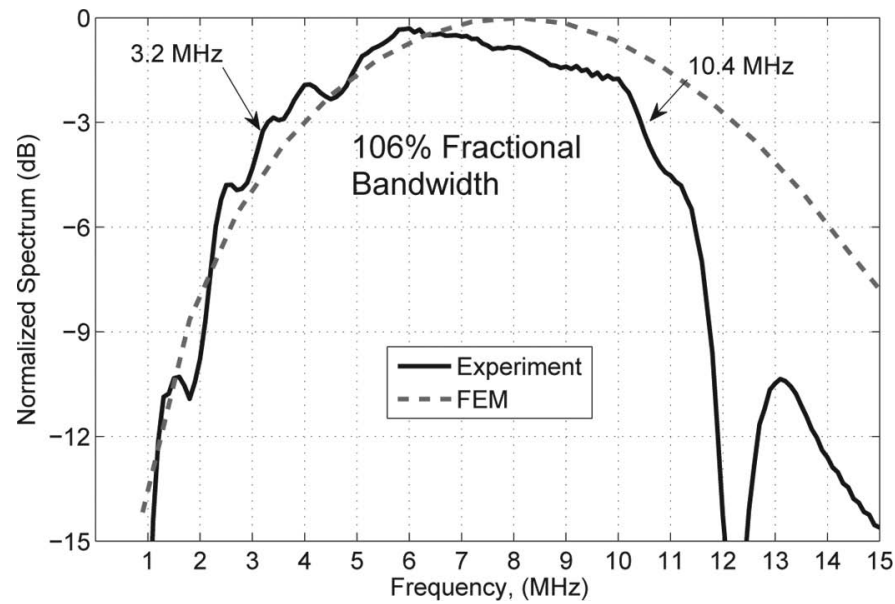

Fig. 11. Normalized transmission spectrum of the CMUTs when excited with 40-ns-long, $-160-\mathrm{V}$ pulse on top of $160-\mathrm{V}$ bias.

able for the collapsed regime. For the sake of achieving a high transmit power, a full electrode coverage is needed.

The calculated electrical and restoring force curves indicate that higher voltages maintain higher forces acting on the membrane for both the collapse and release parts of a unipolar pulse cycle (see Fig. 3). During a negative pulse cycle on top of a bias, first the stored mechanical energy is radiated into the medium as a positive pressure waveform. The membrane is accelerated by high restoring forces and the force acting on the membrane decreases as the membrane is released. Once the membrane transfers its energy to the medium, its velocity drops. Therefore, a damped waveform is transmitted into the medium. However, in the collapse part of the pulse cycle, the electrical forces are low at first and increases with the displacement. Therefore, at the DC stable point, the membrane still has high velocity and kinetic energy, which result in an underdamped waveform and ringing. This behavior is observed in the FEM simulations, as seen in Fig. 10. In the experimental results, the ringing of the membrane is harder to identify because of the substrate ringing, but with careful examination, two harmonics in the first few rings can be identified. The lower-frequency oscillation, which lasts longer, is the substrate ringing with $\sim 80$ ns period. The higher-frequency oscillation is the membrane ringing, which has $\sim 40$ ns period. The approximately $40-$ ns ringing period of the membrane measured in the transmitted pressure waveform in Fig. 10 is consistent with the FEM result.

When the membrane is in contact with the substrate, a charge build-up occurs in the insulation layer. The charging phenomenon is a known problem and has been studied for CMUTs [37]-[39]. The amount of trapped charge in the dielectric layer between the electrodes of the CMUTs increases, especially when the membrane comes in contact with the substrate, because of the increased electric potential across the layer. The control of the amount of charge and the mechanism of charge trapping for micro electromechanical systems devices are still under investigation [40]. In this study, we report achieved experimental re- sults after correcting the applied dc bias voltages with the electric potential generated by the trapped charge in the insulation layer. Initially, a high dc bias voltage $(250 \mathrm{~V})^{3}$ is applied to CMUTs for charging. The electric potential generated by the trapped charge in the insulation layer is determined to be $72 \mathrm{~V}$ by applying a bias voltage until the small signal output pressure reaches its minimum. $72 \mathrm{~V}$ is then used as a correction value for the dc bias voltages reported in this paper.

Recently, CMUTs have been operated without an external dc bias, using the trapped charge in the insulation layer [41]. Such an approach may also be used for deep-collapse operation. However, controlling the amount of charge in the insulation layer in a repeatable manner requires further research.

\section{Conclusions}

This paper describes the mechanics behind the collapsed mode of operation for CMUTs. The static deflection profile of a collapsed membrane was calculated by an analytical approach. Electrical and restoring forces acting on a membrane were calculated for varying membrane displacement. The restoring force increases with increasing bias because of the nonlinear stiffness of the membrane. Similarly, the electrostatic force increases after the collapse because the two electrodes become very close to each other. The analytical results were verified using static FEM simulations. It was demonstrated that the delivered energies during the collapse and release parts of a unipolar pulse cycle increase monotonically with increasing pulse amplitude if the membrane is fully covered with the top electrode.

The transmitted pressure waveform from a CMUT was recorded by a calibrated hydrophone during the immersion experiments. Utilizing higher restoring and electrical forces in the deep-collapse region, we measured $3.5 \mathrm{MPa}$ peak-to-peak pressure with $106 \%$ fractional bandwidth centered at $6.8 \mathrm{MHz}$ on the surface of the transducer for a pulse amplitude of $160 \mathrm{~V}$. The output pressure generated by the deep-collapse regime can be further increased by using full-electrode coverage CMUTs with lower collapse voltages, pulses with higher amplitudes, faster pulse generators, an optimal pulse shape, and elements with higher fill factors.

\section{APPENDIX FEM Simulations}

The finite element simulations are done using ANSYS FEM Package (v12.1, ANSYS Inc., Canonsburg, PA). A 2-D axisymmetric model of CMUT is created. The membrane is modeled with 8 -node structural solid

\footnotetext{
${ }^{3}$ Dielectric breakdown of the silicon nitride film used as the insulation layer is larger than $300 \mathrm{~V}$.
} 
TABle iII. Material Parameters Used in FEM Simulations.

\begin{tabular}{ll}
\hline Material parameters & \\
\hline Young's modulus of $\mathrm{Si}_{3} \mathrm{~N}_{4}, E$ & $110 \mathrm{GPa}$ \\
Poisson's ratio of $\mathrm{Si}_{3} \mathrm{~N}_{4}, \nu$ & 0.27 \\
Dielectric permitivity of $\mathrm{Si}_{3} \mathrm{~N}_{4}, \varepsilon_{\mathrm{r}}$ & 5.4 \\
Density of $\mathrm{Si}_{3} \mathrm{~N}_{4}$ & $3.1 \mathrm{~g} / \mathrm{cm}^{3}$ \\
\hline
\end{tabular}

(PLANE82) elements. Electromechanical transducer elements (TRANS126) were generated under the bottom surface nodes of the membrane using the ANSYS built-in macro EMTGEN. The macro requires a gap value (GAP) to generate ground plane nodes under the selected nodes and creates TRANS126 elements in between. It also performs a point-wise capacitance calculation and provides the necessary inputs for each TRANS126 element. The GAPMIN parameter defines the maximum possible deflection before contact. A contact stiffness factor, $\mathrm{FKN}=1$, is used to overcome convergence problems with a reasonable penetration at the contact interface.

The parameters GAP and GAPMIN are modified to perform a realistic capacitance calculation because TRANS126 elements do not incorporate an insulating layer between the electrodes. The modified parameters are defined as

$$
\begin{aligned}
& \text { GAP }=t_{\mathrm{g}}+\frac{t_{\mathrm{i}}}{\varepsilon_{\mathrm{r}}} \\
& \text { GAPMIN }=\frac{t_{\mathrm{i}}}{\varepsilon_{\mathrm{r}}},
\end{aligned}
$$

where $\varepsilon_{\mathrm{r}}$ is the relative permittivity of the membrane material. The material parameters of the membrane used in the FEM simulations are given in Table III. The effect of atmospheric pressure is included in all simulations.

The dynamic behavior of a CMUT is simulated using the same model with a fluid loading. A fluid column is created over the membrane using 2-D axisymmetric harmonic acoustic fluid (FLUID29) elements. The coupling of structural motion to the fluid pressure at the interface is enabled by specifying fluid-structure flags. The fluid column height is set to a large value, ensuring that there is no reflection from the top boundary at the end of simulation. The height of the fluid column is set to $2 \mathrm{~mm}$. The transient effects are turned off in the first step to ensure a stable membrane under the dc bias. Afterward, transient effects are turned on and the analysis is performed for $1 \mu \mathrm{s}$. The average pressure is captured at $1 \mathrm{~mm}$ above the membrane surface.

\section{REFERENCES}

[1] I. Ladabaum, X. Jin, H. T. Soh, A. Atalar, and B. T. Khuri-Yakub, "Surface micromachined capacitive ultrasonic transducers," IEEE Trans. Ultrason. Ferroelectr. Freq. Control, vol. 45, no. 3, pp. 678 690, 1998.

[2] J. Johnson, Ö. Oralkan, U. Demirci, S. Ergun, M. Karaman, and B. T. Khuri-Yakub, "Medical imaging using capacitive micromachined ultrasonic transducer arrays," Ultrasonics, vol. 40, no. 1-8, pp. 471-476, 2002.
[3] Ö. Oralkan, A. S. Ergun, J. A. Jhonson, M. Karaman, U. Demirci, K. Kaviani, T. H. Lee, and B. T. Khuri-Yakub, "Capacitive micromachined ultrasonic transducers: Next-generation arrays for acoustic imaging?" IEEE Trans. Ultrason. Ferroelectr. Freq. Control, vol. 49, no. 11, pp. 1596-1610, 2002.

[4] R. O. Guldiken, J. Zahorian, F. Y. Yamaner, and F. L. Degertekin, "Dual-electrode CMUT with non-uniform membranes for high electromechanical coupling coefficient and high bandwidth operation," IEEE Trans. Ultrason. Ferroelectr. Freq. Control, vol. 56, no. 6, pp. 1270-1276, 2009.

[5] X. Jin, I. Ladabaum, and B. T. Khuri-Yakub, "The microfabrication of capacitive micromachined ultrasonic transducers," J. Microelectromech. Syst., vol. 7, no. 3, pp. 295-302, 1998.

[6] Y. Huang, A. S. Ergun, E. Hæggström, M. H. Badi, and B. T. Khuri-Yakub, "Fabricating capacitive micromachined ultrasonic transducers with wafer-bonding technology," J. Microelectromech. Syst., vol. 12, no. 4, pp. 128-137, 2003.

[7] J. Knight, J. McLean, and F. L. Degertekin, "Low temperature fabrication of immersion capacitive micromachined ultrasonic transducers on silicon and dielectric substrates," IEEE Trans. Ultrason. Ferroelectr. Freq. Control, vol. 51, no. 10, pp. 1324-1333, 2004.

[8] I. O. Wygant, X. Zhuang, D. T. Yeh, Ö. Oralkan, A. S. Ergun, M. Karaman, and B. T. Khuri-Yakub, "Integration of 2D CMUT arrays with front-end electronics for volumetric ultrasound imaging," IEEE Trans. Ultrason. Ferroelectr. Freq. Control, vol. 55, no. 2, pp. 327-342, 2008.

[9] I. O. Wygant, N. S. Jamal, H. J. Lee, A. Nikoozadeh, Ö. Oralkan, M. Karaman, and B. T. Khuri-Yakub, "An integrated circuit with transmit beamforming flip-chip bonded to a 2-D CMUT array for 3-D ultrasound imaging," IEEE Trans. Ultrason. Ferroelectr. Freq. Control, vol. 56, no. 10, pp. 2145-2156, 2009.

[10] A. Nikoozadeh, Ö. Oralkan, M. Gencel, J. W. Choe, D. N. Stephens, A. de la Rama, P. Chen, K. Themenius, A. Dentinger, D Wildes, K. Shivkumar, A. Mahajan, M. O'Donnell, D. Sahn, and P. T. Khuri-Yakub, "Forward-looking volumetric intercardiac imaging using fully integrated CMUT ring array," in Proc. IEEE Ultrasonics Symp., 2009, pp. 511-514.

[11] F. L. Degertekin, R. O. Guldiken, and M. Karaman, "Annular-ring CMUT arrays for forward-looking IVUS: Transducer characterization and imaging," IEEE Trans. Ultrason. Ferroelectr. Freq. Control, vol. 53, no. 2, pp. 474-482, 2006.

[12] X. Zhuang, D.-S. Lin, Ö. Oralkan, and B. T. Khuri-Yakub, "Fabrication of flexible transducer arrays with through-wafer electrical interconnects based on trench refilling with PDMS," J. Microelectromech. Syst., vol. 17, no. 2, pp. 446-452, 2008.

[13] B. Bayram, Ö. Oralkan, A. S. Ergun, E. Hæggström, G. G. Yaralioglu, and B. T. Khuri-Yakub, "Capacitive micromachined ultrasonic transducer design for high power transmission," IEEE Trans. Ultrason. Ferroelectr. Freq. Control, vol. 52, no. 2, pp. 326-339, 2005.

[14] Ö. Oralkan, B. Bayram, G. G. Yaralioglu, A. S. Ergun, M. Kupnik, D. T. Yeh, I. O. Wygant, and B. T. Khuri-Yakub, "Experimental characterization of collapse-mode CMUT operation," IEEE Trans. Ultrason. Ferroelectr. Freq. Control, vol. 53, no. 8, pp. 1513-1523, 2006.

[15] Y. Huang, E. Hæggstrom, B. Bayram, X. Zhuang, A. S. Ergun, C.-H. Cheng, and B. T. Khuri-Yakub, "Comparison of conventional and collapsed region operation of capacitive micromachined ultrasonic transducers," IEEE Trans. Ultrason. Ferroelectr. Freq. Control, vol. 53, no. 10, pp. 1918-1932, 2006.

[16] R. O. Guldiken, J. McLean, and F. L. Degertekin, "CMUTs with dual-electrode structure for improved transmit and receive performance," IEEE Trans. Ultrason. Ferroelectr. Freq. Control, vol. 53, no. 2 , pp. $483-491,2006$.

[17] S. H. Wong, M. Kupnik, X. Zhuang, D.-S. Lin, K. Butts-Pauly, and B. T. Khuri-Yakub, "Evaluation of wafer bonded CMUTs with rectangular membranes featuring high fill factor," IEEE Trans. Ultrason. Ferroelectr. Freq. Control, vol. 55, no. 9, pp. 2053-2065, 2008.

[18] S. H. Wong, R. D. Watkins, M. Kupnik, K. B. Pauly, and B. T. Khuri-Yakub, "Feasibility of MR-temperature mapping of ultrasonic heating from a CMUT," IEEE Trans. Ultrason. Ferroelectr. Freq. Control, vol. 55, no. 4, pp. 811-818, 2008.

[19] S. H. Wong, M. Kupnik, R. D. Watkins, K. Butts-Pauly, and B. T. Khuri-Yakub, "Capacitive micromachined ultrasonic transducers for therapeutic ultrasound applications," IEEE Trans. Biomed. Eng., vol. 57, no. 1, pp. 114-123, 2010.

[20] A. Lohfink and P. C. Eccardt, "Linear and nonlinear equivalent circuit modeling of CMUTs," IEEE Trans. Ultrason. Ferroelectr. Freq. Control, vol. 52, no. 12, pp. 2163-2172, 2005. 
[21] A. Caronti, G. Caliano, A. Iula, and M. Pappalardo, "An accurate model for capacitive micromachined ultrasonic transducers," IEEE Trans. Ultrason. Ferroelectr. Freq. Control, vol. 49, no. 2, pp. 159-168, 2002.

[22] S. Olcum, M. N. Senlik, and A. Atalar, "Optimization of the gainbandwidth product of capacitive micromachined ultrasonic transducers," IEEE Trans. Ultrason. Ferroelectr. Freq. Control, vol. 52, no. 12, pp. 2211-2219, 2005.

[23] H. Köymen, M. N. Senlik, A. Atalar, and S. Olcum, "Parametric linear modeling of circular CMUT membranes in vacuum," IEEE Trans. Ultrason. Ferroelectr. Freq. Control, vol. 54, no. 6, pp. 1229 $1239,2007$.

[24] A. Bozkurt, F. L. Degertekin, A. Atalar, and B. T. Khuri-Yakub, "Analytic modelling of loss and cross-coupling in capacitive micromachined ultrasonic transducers," in Proc. IEEE Ultrasonics Symp., 1998, pp. $1025-1028$.

[25] G. G. Yaralioglu, A. S. Ergun, and B. T. Khuri-Yakub, "Finite-element analysis of capacitive micromachined ultrasonic transducers," IEEE Trans. Ultrason. Ferroelectr. Freq. Control, vol. 52, no. 12, pp. 2185-2198, 2005.

[26] I. O. Wygant, M. Kupnik, and B. T. Khuri-Yakub, "Analytically calculating membrane displacement and the equivalent circuit model of a circular CMUT cell" in Proc. IEEE Ultrasonics Symp., 2008, pp. $2111-2114$.

[27] A. Caronti, R. Carotenuto, and M. Pappalardo, "Electromechical coupling factor of capacitive micromachined ultrasonic transducers," J. Acoust. Soc. Am., vol. 113, no. 1, pp. 279-288, 2003.

[28] H. K. Oguz, S. Olcum, M. N. Senlik, V. Tas, A. Atalar, and H. Köymen, "Nonlinear modeling of an immersed transmitting capacitive micromachined ultrasonic transducer for harmonic balance analysis," IEEE Trans. Ultrason. Ferroelectr. Freq. Control, vol. 57, no. 2, pp. 438-447, 2010.

[29] B. Bayram, G. G. Yaralioglu, M. Kupnik, A. S. Ergun, Ö. Oralkan, A. Nikoozadeh, and B. T. Khuri-Yakub, "Dynamic analysis of capacitive micromachined ultrasonic transducers," IEEE Trans. Ultrason. Ferroelectr. Freq. Control, vol. 52, no. 12, pp. 2270-2275, 2005 .

[30] S. Timoshenko and S. Woinowsky-Krieger, Theory of Plates and Shells, 2nd ed., New York, NY: McGraw Hill, 1959.

[31] R. L. Pritchard, "Mutual acoustic impedance between radiators in an infinite rigid plane," J. Acoust. Soc. Am., vol. 32, no. 6, pp. 730-737, 1960.

[32] B. Bayram, E. Hæggström, G. G. Yaralioglu, and B. T. KhuriYakub, "A new regime for operating capacitive micromachined ultrasonic transducers," IEEE Trans. Ultrason. Ferroelectr. Freq. Control, vol. 50, no. 9, pp. 1184-1190, 2003.

[33] R. Chanamai and D. J. McClements, "Ultrasonic attenuation of edible oils," J. Amer. Oil. Chem., vol. 75, no. 10, pp. 1447-1448, 1998.

[34] G. S. Kino, Acoustic Waves: Devices, Imaging, and Analog Signal Processing. Englewood Cliffs, NJ: Prentice Hall, 1987.

[35] M. N. Senlik, S. Olcum, H. Köymen, and A. Atalar, "Radiation impedance of an array of circular capacitive micromachined ultrasonic transducers," IEEE Trans. Ultrason. Ferroelectr. Freq. Control, vol. 57, no. 4, pp. 969-976, 2010

[36] A. Bozkurt, I. Ladabaum, A. Atalar, and B. T. Khuri-Yakub, "Theory and analysis of electrode size optimization for capacitive microfabricated ultrasonic transducers," IEEE Trans. Ultrason. Ferroelectr. Freq. Control, vol. 46, no. 6, pp. 1364-1374, 1999.

[37] K. Midtbo and A. Ronnekleiv, "Analysis of charge effects in high frequency CMUTs," in Proc. IEEE Ultrasonics Symp., 2008, pp. 379-382.

[38] H. Martinussen, A. Aksnes, and H. E. Engan, "Investigation of charge diffusion in CMUTs using optical interfermetry," in Proc. IEEE Ultrasonics Symp., 2008, pp. 1218-1221.

[39] S. Machida, S. Migitaka, H. Tanaka, K. Hashiba, H. Enomoto, Y. Tadaki, and T. Kobayashi, "Analysis of the charging problem in capacitive micro-machined ultrasonic transducers," in Proc. IEEE Ultrasonics Symp., 2008, pp. 383-385.

[40] G. Bahl, R. Melamud, B. Kim, S. A. Chandorkar, J. C. Salvia, M. A. Hopcroft, D. Elata, R. G. Hennessy, R. N. Candler, R. T. Howe, and T. W. Kenn, "Model and observations of dielectric charge in thermally oxidized silicon resonators," J. Microeletromech. Syst., vol. 19, no. 1, pp. 162-174, 2010.

[41] K. K. Park, M. Kupnik, H. J. Lee, Ö. Oralkan, and B. T. KhuriYakub, "Zero-bias resonant sensor with an oxide-nitride layer as a charge trap," in IEEE Sensors Conf., 2010, pp. 1024-1028.

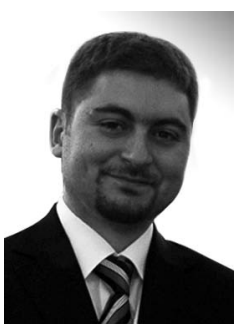

Selim Olçum was born in Chicago, IL, in 1981. He received his B.S., M.S., and Ph.D. degrees in electrical engineering in 2003, 2005, and 2010, respectively, all from Bilkent University, Ankara, Turkey. He worked as a guest researcher at the National Institute of Standards and Technology, Semiconductor Electronics Division, Gaithersburg, MD, during the summers of 2002 and 2003. He was a visiting scholar in the Micromachined Sensors and Transducers Laboratory of the Georgia Institute of Technology, Atlanta, GA, in 2006. He was an instructor in the Electrical and Electronics Engineering Department at Bilkent University for six months in 2011. He is currently a postdoctoral associate in the Department of Biological Engineering and the Koch Institute for Integrative Cancer Research at the Massachusetts Institute of Technology, Cambridge, MA. His dissertation work was focused on developing high-performance micromachined ultrasonic transducers. His current research focus at MIT is to develop real-time techniques for bimolecular detection using micro- and nano-electromechanical devices.

Dr. Olçum was a fellow of ASELSAN during his Ph.D. studies.

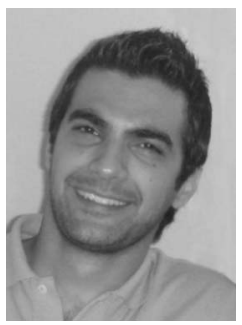

Yalçn Yamaner received his B.Sc. degree from Ege University, Izmir, Turkey, in 2003, and his M.Sc. and Ph.D. degrees from Sabanc University, Istanbul, Turkey, in 2006 and 2011, respectively, all in electrical and electronics engineering. He worked as a visiting researcher at the VLSI Design and Education Center (VDEC), during the summer of 2006. He was a visiting scholar in the Micromachined Sensors and Transducers Laboratory of the Georgia Institute of Technology, Atlanta, GA, in 2008. He is a member of IEEE. His current research interests include micromachined sensors, and transducer modeling and fabrication.

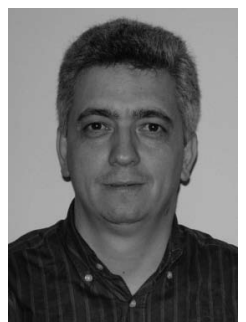

Ayhan Bozkurt received his B.Sc., M.Sc., and Ph.D. degrees from Bilkent University, Ankara, Turkey, in 1992, 1994, and 2000, respectively, all in electrical and electronics engineering. He is currently working as an Associate Professor in the Electronics Engineering Program of the Faculty of Engineering and Natural Sciences of Sabanc University, Istanbul, Turkey. His research interests are transducer modeling and fabrication and frontend integrated circuit design.

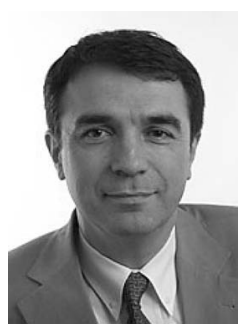

Abdullah Atalar received a B.S. degree from the Middle East Technical University, Ankara, Turkey, in 1974, and M.S. and Ph.D. degrees from Stanford University, Stanford, CA, in 1976 and 1978, respectively, all in electrical engineering. He worked for Hewlett-Packard Labs, Palo Alto, CA, in 1979 . From 1980 to 1986 , he was on the faculty of the Middle East Technical University as an Assistant Professor. In 1986, he joined Bilkent University as the chairman of the Electrical and Electronics Engineering Department and served in the founding of the Department, where he is currently a Professor. In 1995, he was a Visiting Professor at Stanford University. From 1996 to 2010, he was the Provost of Bilkent University. He is presently the Rector of the same university. His current research interests include micromachined devices and microwave electronics.

Prof. Atalar was awarded the Science Award of TUBITAK in 1994. He is a Fellow of IEEE and a member of the Turkish Academy of Sciences. 